\title{
METABOLISMO ÓSEO
}

Póster

Bajos niveles de vitamina $\mathrm{D} y$ su relación con la alteración de lípidos sanguíneos en mujeres posmenopáusicas de 2 municipios del departamento del Atlántico

\section{Becerra JE, Rebolledo RC, Pabón AC, Suárez M, Sarmiento LA.}

Universidad Metropolitana. Barranquilla, Colombia.

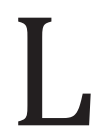

os niveles séricos bajos de vitamina D se han asociado con la alteración de los lípidos sanguíneos, lo cual in-

crementa el riesgo de padecer enfermedades cardiovasculares (ECV). En recientes estudios se ha encontrado una relación entre las bajas concentraciones de vitamina D sanguínea y dislipidemias; sin embargo, se requiere mayor evidencia científica que soporte dicha relación, principalmente en mujeres posmenopáusicas, quienes presentan un mayor riesgo de desarrollar dichas ECV. Este trabajo tuvo como objetivo evaluar los niveles séricos de vitamina D en mujeres posmenopáusicas de los municipios de Malambo y Baranoa, en el departamento del Atlántico, Colombia, y establecer su relación con las concentraciones de lípidos sanguíneos. Para tal fin se realizó un estudio descriptivo, transversal, correlacional, en el que se seleccionó en un muestreo por oportunidad a 87 mujeres posmenopáusicas que asistieron a una institución prestadora de servicios de salud (IPS; PROMOCOSTA) de los municipios mencionados. Se realizó una valoración antropométrica y se tomaron muestras sanguíneas para la evaluación del perfil lipídico y valores séricos de vitamina D. Los resultados mostraron que el 70\% de las mujeres posmenopáusicas evaluadas presentan concentraciones insuficientes/deficientes de vitamina D. Además, se observó que existe una relación estadísticamente significativa $(p<0,05)$ entre los valores bajos de vitamina $\mathrm{D}$ y la alteración del perfil lipídico (altos niveles de triglicéridos y bajas concentraciones de lipoproteína de alta densidad [HDL]). Estos resultados sugieren que se debe incluir la medición de los valores séricos de vitamina D dentro del Plan Obligatorio de Salud (POS) con el fin de suplementar principalmente a las mujeres posmenopáusicas que presenten deficiencias de este micronutriente.

Póster

\section{Déficit de vitamina $\mathrm{D}$ en hombres y mujeres con deseo sexual hipoactivo. Estudio de prevalencia}

\author{
Espitia F, Orozco L.
}

Clínica La Sagrada Familia. Armenia, Colombia.

Objetivo: establecer la prevalencia del déficit de vitamina D en hombres y mujeres con deseo sexual hipoactivo.

Metodología: estudio observacional, descriptivo, de corte transversal, realizado desde mayo de 2011 a noviembre de 2017 en Armenia, Quindío, Colombia. Se reclutó, de forma consecutiva, una muestra de 107 pacientes diagnosticados con deseo sexual hipoactivo. El muestreo fue probabilístico por conveniencia y consecutivo. Se incluyeron hombres y mujeres mayores de edad. Se excluyeron analfabetas, gestantes, pacientes que presentaban enfermedad neoplásica, mujeres con menopausia quirúrgica, hombres prostatectomizados, padecimientos metabólicos, trastornos mentales o déficit mental. Los resultados se exponen de manera agrupada para el total de la población.

Resultados: la prevalencia del déficit de vitamina $\mathrm{D}$ fue del $63,55 \%$, de los cuales el 34,57 \% eran hombres y el 28,97 \% eran mujeres. Los niveles de vitamina D fueron inferiores en los hombres con respecto a los de las mujeres $(34,57$ frente a 41,36, $p$ 
$<0,001)$, con mayor porcentaje de deficiencia $(21,49$ frente a 14,95 $\%, p<0,007)$ y de insuficiencia $(16,82$ frente a $10,28 \%, p<0,001)$.

Conclusión: es considerable la prevalencia del déficit de vitamina $\mathrm{D}$ en hombres y mujeres con deseo sexual hipoactivo. Se hace necesaria la cuantificación de sus niveles con el fin de establecer la suplencia como parte del tratamiento.

Palabras clave: disfunciones sexuales fisiológicas, diagnóstico, hombres, mujeres, prevalencia, vitamina D.

\section{Póster}

\section{Determinación del consumo diario de calcio en la población colombiana}

Arenas HM, Arias D, López VJ, Arias DR.

Clínica Comfamiliar Risaralda. Pereira, Colombia.

Introducción: existe una fuerte asociación entre la baja ingesta de calcio y la incidencia de osteoporosis. En Colombia son escasos los estudios realizados en los que se valoren los niveles de calcio ingeridos por la población de acuerdo con el sexo, edad, estrato socioeconómico, nivel educativo y comorbilidades asociadas. La ingesta diaria recomendada (RDI) de calcio la establecen organismos internacionales con el fin de promover una alta densidad mineral ósea (DMO) en la población. Se requiere conocer la ingesta promedio de calcio de los colombianos para determinar el riesgo y promover estrategias de prevención.

Objetivo: estimar y comparar la ingesta promedio de calcio y el consumo recomendado en personas adultas entre $18 \mathrm{y}$ 60 años de acuerdo con el sexo, edad, estrato socioeconómico, nivel educativo, estado de osteopenia-osteoporosis y uso de suplementos de calcio.

Metodología: estudio observacional descriptivo, realizado en varios centros de Colombia, con población masculina y femenina mayor de 18 años. Se aplicó un instrumento validado por la International Osteoporosis Foundation (IOF) para estimar el consumo diario de calcio y se comparó con la ingesta recomendada de calcio en el grupo poblacional según los parámetros internacionales.

Resultados: se aplicó el instrumento a 179 pacientes, de los cuales $60,9 \%$ fueron de sexo femenino. El consumo promedio de calcio fue $601 \mathrm{mg} /$ día sin diferencias entre hombres o mujeres. Respecto a los grupos etarios, las personas en edades entre 35-44 años tuvieron mayor consumo de calcio. Hubo una relación directamente proporcional entre el nivel socioeconómico y el promedio de consumo de calcio. Cuando se comparó el consumo promedio de calcio con la ingesta recomendada, se determinó que el 81,5 \% de la población no cumplía con el requerimiento de calcio recomendado al día.

\section{Póster}

\section{Efficacy of Teriparatide Compared with Risedronate on $\operatorname{Frax}^{\circledR}$ - Defined Major Osteoporotic Fractures: a Post-Hoc Analysis of the VERO Clinical Trial}

\author{
Body $J^{1}$, Marin $F^{2}$, Geusens $P^{3}$, Zerbini $C^{4}$, Fahrleitner- \\ Pammer $A^{5}$, Moericke $R^{6}$, et al. \\ ${ }^{1} \mathrm{CHU}$ Brugmann, Université Libre de Bruxelles (ULB), Brussels, Belgium \\ ${ }^{2}$ Lilly Research Center Europe, Madrid, Spain \\ ${ }^{3}$ Maastricht University Medical Center, Maastricht, The Netherlands \\ ${ }^{4}$ Centro Paulista de Investigaçao Clínica, Sao Paulo, Brazil \\ ${ }^{5}$ Division of Endocrinology, Medical University of Graz, Graz, Austria \\ ${ }^{6}$ Institut Präventive Medizin \& Klinische Forschung, Magdeburg, Germany.
}

Objectives: the VERO, a 24-month double-blind trial, recruited osteoporotic postmenopausal women and reported significant relative risk (RR) reduction in clinical fractures (CFx) with teriparatide (TPTD) compared with risedronate (RIS). This post-hoc analysis of the VERO trial was restricted to the major osteoporotic fractures (MOF; i.e. clinical vertebral, hip, humerus and forearm), as defined by FRAX ${ }^{\circledR}$.

Methods: a total of 1360 postmenopausal women (mean age $72.1 \pm 8.7$ years; $\geq 2$ moderate or 1 severe VFx and BMD

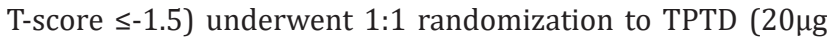
daily, SC) or RIS (35 mg weekly, oral) treatments. We calculated the cumulative incidence of patients and treatment comparison based on Kaplan-Meier and stratified log-rank tests, adjusted for clinical vertebral fracture $\leq 12$ months before randomization and bisphosphonate use. Censorship was applied to data from patients lost to follow-up, deceased, or without any fracture during the study.

Results: incidence of $\geq 1$ low-trauma MOF was significantly lower in the group treated with TPTD compared with those who received RIS (2.6 \% vs. $6.4 \%$; hazard ratio [HR]: 0.40; $95 \%$ confidence interval [CI]: 0.23-0.68; $p=0.001$; absolute RR: $3.8 \%$ ). For TPTD and RIS, clinical vertebral (7 and 24) and radius ( 6 and 10) were the most common types of CFx. The group treated with RIS experienced hipper CFx than those treated with TPTD (5 vs. 2); whereas those in the TPTD group suffered more humerus CFx than those in the RIS group (4 vs. 
2). The incidence of clinical vertebral fractures was $1.1 \%$ and $3.9 \%$ with TPTD and RIS, respectively (HR: 0.29; $95 \% \mathrm{CI}$ : 0.14-0.58; $p=0.002$ ).

Conclusion: in postmenopausal women with severe osteoporosis, administration of TPTD resulted in greater efficacy rates than administration of RIS, with a $60 \%$ reduction of FRAX ${ }^{\circledR}$-defined MOFs.

Declaration of funding sources: Funded by Eli Lilly and Company.

Original work presented at the $40^{\text {th }}$ Annual Meeting of the American Society for Bone and Mineral Research (ASBMR 2018). Presentation \#902. Available at http://www.asbmr. org/education/2018-abstracts.

\section{Póster}

\section{Osteoporosis y baja masa ósea en mediciones de densitometría en Villavicencio, Meta, durante el 2018}

Rosero FO, Garcés H, Beltrán A, Guerrero JF, Escobar $L M$, Galeano D, et al.

Instituto de Osteoporosis de los Llanos. Villavicencio, Colombia.

Introducción: la osteoporosis se define como un trastorno esquelético caracterizado por un compromiso en la fortaleza del hueso y una predisposición aumentada en el riesgo de fractura.

El método de diagnóstico de elección es la absorciometría con rayos X de energía dual (DXA). El criterio para definir y diagnosticar osteoporosis en mujeres posmenopáusicas es el hallazgo de un T-score menor o igual a -2,5 en la columna lumbar, cuello femoral, cadera o radio. El hallazgo de un T-score entre $-1,0$ y $-2,5$ en la columna lumbar, cuello femoral, cadera o radio se considera osteopenia.

Materiales y método: se revisaron los registros de densitometrías tomadas durante el período de enero a diciembre de 2018 en el Instituto de Osteoporosis de los Llanos en la ciudad de Villavicencio y se analizaron los datos en Excel.

Resultados: durante el período de enero a diciembre de 2018 se realizó un total de 2012 mediciones de DXA; de estas, el 95 \% se practicó a hombres y mujeres mayores de 50 años. Se registró el diagnóstico de osteoporosis en el 42,7 \% de las mediciones, osteopenia en el 39,7 \% y normal en el 12,6 \% de los estudios realizados (Figura 1).
Figura 1. Resultados de DXA en la población estudiada.

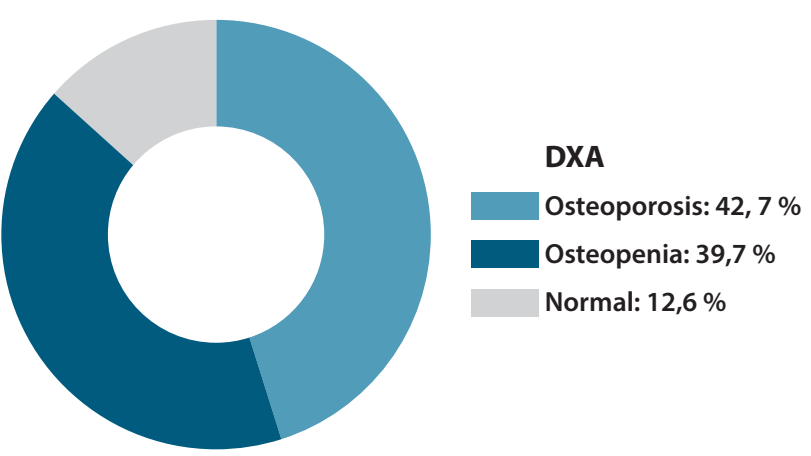

Discusión: aunque se trató de una medición con demanda inducida ya que en su mayoría son pacientes remitidos para el examen por orden médica y no como parte de un programa de tamizaje a la población general, llama la atención el elevado número de pacientes con osteoporosis y osteopenia, por encima del reportado en otros estudios realizados en el mundo y en nuestro país, como el realizado en el Hospital Central de la Policía Nacional, en 2072 densitometrías, con el 95\% de ellas realizadas en mujeres de entre 50 y 70 años, que mostró osteoporosis en cualquier localización en el 32 \% y osteopenia en el $42 \%$ de las mujeres analizadas.

Este estudio deja abierta la discusión sobre la necesidad de realizar estudios de tamizaje para determinar la verdadera prevalencia de osteoporosis en nuestro país.

\section{Lecturas recomendadas}

- Medina Orjuela A, Rosero Olarte O, Rueda Plata PN, et al. II Consenso Colombiano para el Manejo de la Osteoporosis Posmenopáusica. Rev Colomb Reumatol. 2018;25(3):184-210.

- $\quad$ Morales-Torres J, Gutiérrez-Ureña S. The burden of osteoporosis in Latin America. Osteoporos Int. 2004;15(8):625-32.

- Páez A. Densitometría ósea: utilidad diagnóstica. Revista del Hospital Central de la Policía Nacional, 1999:2:27-32.

\section{Póster}

\section{Osteoporosis, sarcopenia y correlación entre SARC-F y masa muscular por DXA en población colombiana: estudio piloto}

\author{
Castrillón MP, Flórez AM, Vergara JE, Roa A, \\ Quintero J, Parra G, et al.
}

Universidad Autónoma de Bucaramanga, Clínica Fundación Oftalmológica de Santander (FOSCAL). Bucaramanga, Colombia. 
Objetivo: determinar la prevalencia de osteosarcopenia en ancianos colombianos y la correlación del cuestionario SARC-F con baja masa muscular por absorciometría con rayos $\mathrm{X}$ de energía dual (DXA).

Métodos: estudio de corte trasversal en mayores de 60 años enviados a densidad mineral ósea (DMO) de tamizaje o seguimiento de osteoporosis en radiólogos especializados de FOSCAL entre abril y noviembre de 2018. Se aplicó el cuestionario SARC-F y la prueba short physical performance battery (SPPB). La presencia de sarcopenia se determinó a través de los criterios europeos European Working Group on Sarcopenia in Older People 2 (EWGSOP 2) y la osteoporosis por un valor T menor de -2,5 según la Organización Mundial de la Salud (OMS).

Resultados: la población estudiada fue de 61 pacientes con una edad promedio de 73,2 años (64-89), el $91 \%$ era de sexo femenino y el índice de masa corporal (IMC) en promedio fue de 27,6. El $26 \%$ tenía fracturas previas, el 28,5 \% presentó un SARC-F $\geq 4,37$ y el $5 \%$ de los pacientes presentó osteoporosis. El promedio del índice de masa muscular esquelética (IMME) fue de $6,78 \mathrm{~kg} / \mathrm{m}^{2}(5,24-8,03)$. El $22 \%$ de los pacientes tenía una masa muscular baja según los criterios EWGSOP 2 $\left(<7 \mathrm{~kg} / \mathrm{m}^{2}\right.$ en hombres y $<6 \mathrm{~kg} / \mathrm{m}^{2}$ en mujeres). La prevalencia de osteosarcopenia fue del 12,5\% (osteopenia/osteoporosis más sarcopenia [SARC-F $>4$ ] y baja masa muscular). Solo el 15 $\%$ de los pacientes con SARC-F $>4$ tenía baja masa muscular.

Conclusión: este es el primer estudio que evalúa la osteosarcopenia por DXA en Colombia. El SARC-F presenta una baja correlación con la masa muscular esquelética. Aunque el EWGSOP 2 recomienda la detección de sarcopenia con SARC-F, es importante evaluar la composición corporal y la toma de medidas del rendimiento físico (SPPB) para el diagnóstico.

\section{Póster}

\section{Patient Characteristics and} Fracture Outcomes in Patients Previously Treated with Bisphosphonates or TreatmentNaïve in The Teriparatide Versus Risedronate VERO Clinical Trial

\author{
Hadji $P^{1}$, Marin $F^{2}$, Kendler $D L^{3}$, Geusens $P^{4}$, Russo $L^{5}$, \\ Malouf $J^{6}$, et al.
}

${ }^{1}$ Krankenhaus Nordwest GHMB, Frankfurt, Germany.

${ }^{2}$ Lilly Research Center Europe, Madrid, Spain.

${ }^{3}$ University of British Columbia, Vancouver, Canada.

${ }^{4}$ Maastricht University Medical Center, Maastricht, The Netherlands.

${ }^{5}$ Centro de Analises e Pesquisas Clínicas LTDA, Rio de Janeiro, Brazil.

${ }^{6}$ Hospital Sant Pau, Barcelona, Spain.

Objectives: in the VERO trial, teriparatide (TPTD) lowered the risk for new vertebral and clinical fractures (VFx and CFx, respectively) versus risedronate (RIS), in both bisphosphonate (BP) users and treatment-naïve patients. Here, we report a post-hoc analysis comparing baseline patient characteristics, by prior BP therapy.

Methods: The VERO study population (1360 postmenopausal women with $\geq 2$ moderate or 1 severe VFx and BMD T-score $\leq-1.5$ ) was randomized 1:1 to subcutaneous $20 \mu \mathrm{g} /$ day TPTD or oral RIS at weekly doses of $35 \mathrm{mg}$. Patients were further grouped as either BP users (oral, intramuscular [IM] or intravenous [IV] BP for $\geq 3$ months; or $\geq 1$ IV dose of zoledronic acid) and treatment-naïve (either none or $<3$ months of osteoporosis treatment). Calcium and vitamin D supplements were not considered osteoporosis medications. Non-BP user data $(\mathrm{n}=57.4 \%)$ are not presented.

Results: overall, $53.5 \%$ of VERO patients were former BP users, while $42.3 \%$ were treatment naïve. Former BP users were older, had lower body mass indexes (BMI), reported non-VFx with greater frequency, and were predominantly from Europe and North America ( $p<0.01$ for all comparisons). Compared against treatment-naïve patients, former BP users had significantly greater 25(OH)D levels (33.3 vs $29.7 \mathrm{ng} / \mathrm{mL}$; $p<0.01)$ and fewer recent VFx $(32.0 \%$ vs $43.0 \%$; $p<0.0001)$. Prevalence and severity of VFx, BMD T-scores and number of patients with $\geq 1$-hip fractures or glucocorticoid therapy were similar in both groups.

Conclusion: BP-treated and treatment-naïve patients enrolled in the VERO trial had relatively similar characteristics. Differences in baseline characteristics among groups were unlikely to have impacted on the reduction of fracture risk observed in women randomized to either TPTD or RIS.

Declaration of funding sources: Funded by Eli Lilly and Company.

The original work was presented at the $40^{\text {th }}$ Annual Meeting of the American Society for Bone and Mineral Research (ASBMR 2018). Presentation \#926. Available at http://www. asbmr.org/education/2018-abstracts. 
Póster

\section{Prevalencia del déficit de vitamina $D$ y factores de riesgo asociados en gestantes del Quindío}

\author{
Espitia F, Orozco L.
}

Clínica La Sagrada Familia. Armenia, Colombia.

Objetivo: determinar la prevalencia del déficit de vitamina D y factores de riesgo asociados en gestantes del Quindío.

Metodología: estudio de corte transversal descriptivo y prospectivo, de muestreo consecutivo en 2 centros de atención de la ciudad de Armenia entre mayo de 2014 y agosto de 2017. La población de estudio incluyó a 504 gestantes.

Resultados: la edad promedio fue de 24,8 \pm 15,9 años, con un rango entre 18 y 45 años. La mediana de la edad gestacional fue de 27 semanas (rango entre 9 y 42 semanas). La prevalencia del déficit de vitamina $\mathrm{D}$ en el grupo estudiado estuvo presente en el 32,14 \% de mujeres; la insuficiencia fue la de mayor prevalencia con el 23,41\%, mientras que la deficiencia reportó el $8,73 \%$. En el primer trimestre de embarazo se observó una prevalencia del 23,45 \%; en el segundo trimestre, de 35,18\%; y en el tercer trimestre, de 41,35\%. Se encontraron como factores de riesgo para el déficit el uso excesivo protectores solares (Odds ratio [OR]: 5,7; intervalo de confianza [IC] $95 \%$ : 2,91-9,84), la presencia de baja concentración de vitamina D en los alimentos consumidos (OR: 3,14; IC 95 \%: 1,79-6,43), inadecuada exposición a la luz solar (OR: 4,81; IC 95 \%: 2,92-7,65) y sobrepeso y obesidad (OR: 3,76; IC 95 \%: 1,89-6,54).

Conclusión: en las mujeres gestantes del Quindío existe una prevalencia de déficit de vitamina D cercana a la tercera parte, caracterizada principalmente por insuficiencia de 25(OH)D3, lo cual podría tener repercusiones negativas en la salud maternofetal. Se requieren prontas y oportunas intervenciones para establecer un plan diagnóstico y terapéutico inmediato.

Palabras clave: deficiencia de vitamina D, factores de riesgo, mujeres embarazadas, dieta.

\section{Póster}

\section{Prevalencia del déficit de vitamina D en mujeres posmenopáusicas de Armenia}

\author{
Espitia F, Orozco Santiago L.
}

Objetivo: determinar la prevalencia del déficit de vitamina D en mujeres posmenopáusicas en Armenia, Quindío.

Materiales y métodos: estudio de corte transversal descriptivo y prospectivo en un centro de atención de la ciudad de Armenia, realizado entre octubre de 2012 y septiembre de 2018 . Se incluyó una población de 537 mujeres posmenopáusicas atendidas en la consulta de menopausia y climaterio, mayores de 40 años de edad, con diagnóstico de menopausia (hormona foliculoestimulante $[\mathrm{FSH}]>40 \mathrm{mlU} / \mathrm{L}$ o 12 meses de amenorrea). Se excluyeron pacientes con menopausia iatrogénica y las que no quisieron participar. Se les determinó la concentración plasmática de 25(OH) D3 mediante cromatografía líquida de alta resolución. Se definió la deficiencia con rangos de 25(OH)D3 $<20 \mathrm{ng} / \mathrm{mL}$ e insuficiencia 20-29,9 ng/mL. La información recolectada se describió y analizó mediante técnicas estadísticas convencionales, con análisis estratificado según las variables.

Resultados: la edad promedio fue de 53,7 \pm 8,7 años (rango: 42 a 78). La concentración media en la población total de 25(OH)D3 fue 28,5 ng/mL (rango: 7,5-80,4). La prevalencia del déficit de vitamina $\mathrm{D}$ en el grupo estudiado estuvo presente en el 73,92 \% ( $n=397)$ de las mujeres, las cuales reportaron valores $<30 \mathrm{ng} / \mathrm{mL}$, una prevalencia de insuficiencia del 55,16\% (n = 219) y de deficiencia del 44,83\% $(n=178)$.

Conclusiones: se detectó que alrededor del $75 \%$ de las mujeres posmenopáusicas presenta niveles de vitamina D dentro de los parámetros establecidos para deficiencia o insuficiencia, lo cual se constituye en un problema de salud pública. Se hace necesario mejorar la eficiencia e impacto de los programas de suplementación con vitamina D en este grupo poblacional.

Palabras clave: avitaminosis, deficiencia de vitamina D, posmenopausia, mujeres, prevalencia.

\section{Póster}

Relación entre el índice TG/ HDL-C, la resistencia a la insulina y la vitamina $\mathrm{D}$ sérica en mujeres posmenopáusicas del departamento del Atlántico, Colombia

Sarmiento $L A^{1}$, Becerra JE $E^{1}$, Rebolledo $R^{2}$, Suárez $M^{1}$, Barragán $M^{1}$, Sánchez $L^{1}$.

${ }^{1}$ Programa de Nutrición y Dietética, Universidad Metropolitana. Barranquilla, Colombia.

${ }^{2}$ Programa de Fisioterapia, Universidad Metropolitana. Barranquilla, Colombia.

Clínica La Sagrada Familia. Armenia, Colombia. 
$\mathrm{L}$ a relación triglicéridos (TG)/lipoproteínas de alta densidad (HDL-C) es un indicador de riesgo cardiovascular (RC) de importante valor pronóstico. Sin embargo, la relación entre el índice TG/HDL-C y otros parámetros de RC como la resistencia a la insulina (RI) determinada por el modelo homeostático para evaluar la resistencia a la insulina (HOMA-IR) y la deficiencia de vitamina D sérica se ha documentado poco en la mujer posmenopáusica.

Objetivo: determinar la relación entre el índice TG/HDL-C, el índice HOMA como marcador de insulinorresistencia y los niveles séricos de vitamina $\mathrm{D}$ en mujeres posmenopáusicas del departamento del Atlántico.

Métodos: diseño descriptivo transversal correlacional en el que se vincularon por muestreo de oportunidad $183 \mathrm{mu}-$ jeres posmenopáusicas concurrentes a una entidad de salud. Se evaluaron la glucemia basal, perfil lipídico, insulina sérica (kit LIAISON-inmunoquimioluminiscencia), vitamina D (LIAISON-25(OH)Vitamina D total inmunoquimioluminiscencia) en condición de ayuno. La resistencia a la insulina se determinó con el modelo matemático HOMA-IR (ecuación: glucemia [mg/ $\mathrm{dL}] / 18 \times$ insulinemia [microU/mL]/22,5).

Resultados: el índice TG/HDL-C fue elevado $(\geq 3,0)$ en el $56,83 \%$ de las mujeres y el HOMA-IR fue $\geq 2,5$ en el 53,01 $\%$. La correlación lineal de estos 2 índices fue significativa ( $p$ $<0,05)$. La condición de deficiencia o insuficiencia de vitamina D sérica se relacionó directa y significativamente con el índice TG/HDL-C ( $p=0,007$, prueba de análisis de la varianza [ANOVA]), pero no con el HOMA-IR.

Conclusión: la relación TG/HDL-C con la RI determinada por HOMA-IR y con la deficiencia de vitamina D fue significativa en mujeres posmenopáusicas del departamento del Atlántico, Colombia.

\section{Póster}

\section{Tabaquismo y vitamina D: una vía a la fragilidad}

Pérez $M U^{1,2}$, Patiño $D^{1,3}$, Borda $M G^{1,4}$.
${ }^{1}$ Semillero de Neurociencias y Envejecimiento e Instituto de Envejecimiento, Pontificia Universidad Javeriana. Bogotá D. C., Colombia.

${ }^{2}$ Geriatric Epidemiology Research Unit, Instituto Nacional de Geriatría. México D. F., México.

${ }^{3}$ Departamento de Medicina Interna. Hospital Universitario San Ignacio. Bogotá D. C., Colombia.

${ }^{4}$ Centre for Age-Related Diseases, Stavanger University Hospital. Stavanger, Noruega.

Introducción: el tabaquismo es responsable de alrededor de 6 millones de muertes al año, la mayor parte de estas de forma prematura. Los adultos mayores parecen ser menos conscientes de los riesgos que conlleva y son menos propensos a la cesación. Así mismo, el déficit de vitamina D y la fragilidad se asocian con múltiples desenlaces adversos.

Métodos: el presente es un estudio de casos y controles dentro en una cohorte con datos derivados del Estudio Nacional de Salud y Envejecimiento en México (ENASEM). Se categorizaron las variables del siguiente modo: bioquímicos (vitamina D y proteína C-reactiva), geriátricos (fragilidad y caídas) y uso de servicios médicos (citas médicas y hospitalizaciones en el año previo). La exposición se dividió en quienes nunca fumaron (referencia), cesación tabáquica y tabaquismo actual. Se realizaron análisis multivariados ajustados por variables de confusión.

Resultados: la muestra se compone de 1102 sujetos $\geq 60$ años. El 27,68 \% había fumado en algún momento y el 11,16 $\%$ correspondía a fumadores activos. Se encontró fragilidad en el 42,06 \% de la muestra, el 37 \% tenía hipovitaminosis D y el 11,52 \% había sido hospitalizado el año anterior. La regresión logística multivariada reveló una asociación entre tabaquismo e hipovitaminosis D (Odds ratio [OR]: 3,01; intervalo de confianza [IC] $95 \%$ : 1,55-5,83; $p=0,01$ ).

Discusión: la deficiencia de vitamina D se asocia con el tabaquismo. Nuestros resultados revelaron la importancia de la cesación tabáquica aun en sujetos ancianos y suplencia vitamínica en caso de hipovitaminosis con el fin de evitar la fragilidad y los desenlaces adversos asociados, y mejorar la calidad de vida en dicha población. 